\title{
Curvature-Constrained Motion Within a Limited Workspace*
}

\author{
Andrei M. Shkel and Vladimir J. Lumelsky \\ University of Wisconsin-Madison \\ Madison, Wisconsin 53706, USA
}

\begin{abstract}
We pose the following questions: Given two points within a closed area $W \subset \mathbb{R}^{2}$, each with a prescribed direction of motion in it, (i) what is the shortest path of bounded curvature that joints them and lies completely in W? (ii) what is the minimum number of cusps one needs to design a path in W? This kind of questions appear in various applications, such as robot motion planning. The proposed approach makes use of a tool dubbed the Reflective Unfolding operator which has a clear geometric interpretation and provides an interesting means for solving other trajectory design problems. In this text, the approach is applied to the following problem: for a car moving with bounded curvature and possible reversals, given the starting and target directions of motion at the center of a disc $D$ of some radius $R$, design the shortest possible path fully lying in $D$. The path produced by the algorithm turns out to also be of the lowest complexity (the minimum number of cusps).
\end{abstract}

\section{INTRODUCTION}

We address the question of finding the shortest path for a car with nonholonomic constraints. The car can move only along paths of bounded curvature, not more than $1 / \rho_{\min }$. The path is to connect the initial and final points, $q_{0}, q_{f}$, in $\mathbb{R}^{2}$, each with a specified orientation angle (direction of motion), $\phi_{0}$ and $\phi_{f}$. Together, coordinates $p=(q, \phi)$ are called a configuration. For the case of unlimited operational space, the complete solution to this problem was given by J. Reeds and L. Shepp [1]. We consider a more general case when the path between the two configurations must lie within a closed domain $\mathbf{W} \subset \mathbb{R}^{2}$.

The existence of a path between two configurations which are in the same open component of free space was shown by J.P. Laumond [2]: The idea of the proof is to approximate the path by a sequence of short back and forth motions (reversals). As mentioned in [3], such approximation may include very many reversals, and so it

\footnotetext{
*'This work was supported by the National Oceanic and Atmospheric Administration, US Dept of Commerce, grant NA46RG048.
}

would be of interest to find a motion that minimizes the number of reversals.

For the case with no constraints on the car's environment, Dubins [4] showed how to compute the shortest paths with forward (smooth) motion only. This result was later extended by Reeds and Shepp [1] to the optimal solution for a more complex case with motion reversals (cusps). The case left open by these works - obtaining solutions for a constrained environment - is very important for applications, such as robot motion planning.

In partially known environment (sensor-based planning) the situation with obstacles can be reduced to that of motion in a limited space without obstacles, by taking an area around the robot that is free of obstacles and then designing a path inside of this area. A related question here is to find the shortest path or the path with the fewest reversals. For the real-time version of robot motion planning, the said area is defined at every step of the path based on sensing information; then, a point on this area boundary that satisfies some global planning considerations is defined as an intermediate target, the shortest path toward this target is defined, and a physical step along this path is made; then the sequence repeats [5].

A strategy for finding the shortest path in a limited area is the subject of this work. We introduce a new tool called the Reflective Unfolding operator (RU-operator) which maps the original problem of planning a path with reversals in a limited area into an equivalent problem of planning a smooth path in an unlimited space. A successive application of the RU-operator yields the optimal solution. The technique can be used for a variety of shortest path problems. In the spirit of works $[1,2,3,4]$, we assume that the car's path curvature is constrained, may include reversals of motion, and is confined to a disc-shaped area $D$ of radius $R$.

Assume for the moment that the path does already exist. Order all its reversal cusps sequentially, starting at the initial configuration. In a single application of the RU-operator, it will take two subpaths adjoining the first cusp, and "unfold" the second subpath so as to produce a smooth cuspless piece of circle around the former cusp 
point, while preserving the original tangent to both segments. The next cusp is then treated in a similar fashion, thus producing out of path segments a large circular arc of radius $\rho$, with multiple discs of radius $R$ superimposed on it (see Section IV and Figure 4).

The operator possesses a number of properties that make it a good tool for calculating optimal paths. For example, the car's final orientation defines uniquely a tangent line to the circle $\rho$, and so from the distance (along that circle) between the initial and final configurations one can quickly calculate the number of segments (i.e. the total length of) in the optimal path, even without calculating the actual path.

In this text, the development and application of the RU-operator and the control strategy for generating the optimal path is illustrated on a special case where only the orientation angles of the initial and final configurations differ. Some proofs omitted due to space limitations.

\section{Problem Statement; Notations}

The domain $\mathbf{W} \subset \mathbb{R}^{2}$ in which the car operates is a disc, $D\left(q_{0}, R\right)$, with center $q_{0}$ and radius $R$. The car's orientation is associated with its front end and defined by the orientation angle, $\phi$, measured from the $x$-axis to the orientation line perpendicular to the wheel axes and passing through the center of mass, Figure 1. The radius of car's turning cannot be less than a specified value, $\rho_{\min }$.

The control inputs responsible for forward or backward motion are considered in terms of the car translational velocity and angular (steering) velocity. Let $q=(x, y)$ denote the coordinates of the car's center of mass $C$ in $\mathbf{W}$; $\rho=\rho(q)$ is the radius of curvature at $q ; v$ - the magnitude of translational velocity of $C$; and $\omega$ - the magnitude of angular velocity. We assume a unit translational velocity, $v= \pm 1$, and a limit on angular velocity, $1 / \rho_{\min }$ radian per time unit. Assume that the wheels do not slide; that is, the velocity of the car's center of mass coincides with the orientation line and is tangent to the path. This assumption imposes a nonholonomic constraint on the car motion, of the form $\dot{x} \sin \phi-\dot{y} \cos \phi=0$.

The car kinematics is modeled as follows:

$$
\dot{x}=v \cos \phi, \quad \dot{y}=v \sin \phi, \quad \dot{\phi}=\omega .
$$

The task thus is: Given the car's initial and final configurations, $p_{0}=\left(0,0, \phi_{0}\right)$ and $p_{f}=(0,0,0)$ (that is, both positions $q_{0}, q_{f}$ are at the origin $O$, and $\phi_{f}=0$ ), find the shortest possible path for the car's center of mass $C$ that lies fully inside the disc $D$.

Phase space. At an instant $t$, the state of the car is completely specified by its position $q_{t}=\left(x_{t}, y_{t}\right)$ and velocity $v_{t}=\left(\dot{x}_{t}, \dot{y}_{t}\right) ; t$ can also be interpreted as a running parameter, e.g. an arclength. The car motion is confined

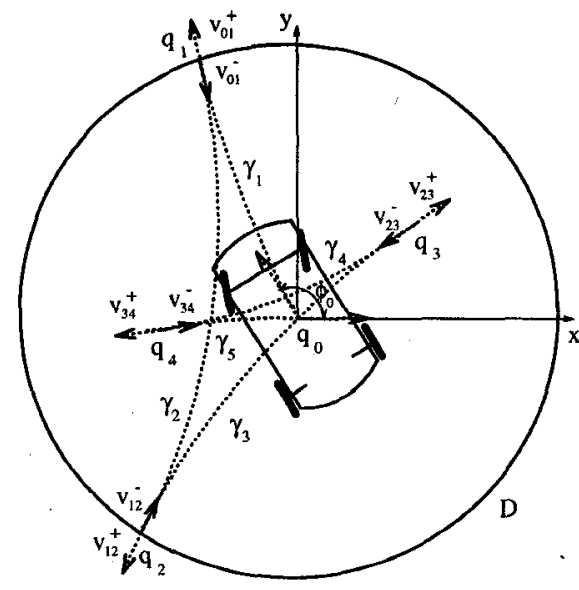

Fig. 1. From its initial configuration $\left(q_{0}, \phi_{0}\right)$, the car is to arrive at the final configuration $\left(q_{0}, 0\right)$, along the shortest path possible and so that its center of mass is confined to the disc $D$. The resulting path here is made of five arc segments and has four cusps, $q_{0} \rightarrow q_{1} \rightarrow q_{2} \rightarrow q_{3} \rightarrow q_{4} \rightarrow q_{0}$.

to the closed domain $\mathbf{W}$ with the boundary $\Gamma=\partial \mathbf{W}$ :

$$
\mathbf{W}=\left\{q \in \mathbb{R}^{2}: f(q) \geq 0\right\}, \Gamma=\left\{q \in \mathbb{R}^{2}: f(q)=0\right\}
$$

In our case, $\mathbf{W}$ is the disc $D\left(q_{0}, R\right)$, and $\Gamma$ is its boundary circle of radius $R$. Both $\mathbf{W}$ and $\Gamma$ are specified by the function $f(x, y)=R^{2}-x^{2}-y^{2}$.

Denote by $\mathbf{P}$ the unit tangent bundle over $\mathbf{W}$. The points of $\mathbf{P}=\mathbf{W} \times\left\{S^{1}=[0,2 \pi]\right\}$ are of the form $p=$ $(q, \phi)$, where $q \in \mathbf{W}$ and $\phi \in S^{1}$ specify the tangent to the path at points of $\mathbf{W}$. The direction of the tangent vector coincides with the direction of motion; it can be identified with the unit velocity vector $v$. The pair $(q, v)$, which is a point in the three-dimensional phase space $\mathbf{P}$, uniquely determines the car position on the path. Function $p(t)=$ $(q(t), v(t))$ defines the whole car trajectory.

Suppose $\pi: \mathbf{P} \rightarrow \mathbf{W}$ is the natural projection for $p=(q, v) \in \mathbf{P}$; i.e. $\pi(q, v)=q$ and $\mathbf{P}=\pi^{-1}(\mathbf{W})$. Generally speaking, if $\mathbf{W}$ is a compact Riemann manifold with piecewise smooth boundary, then $\mathbf{P}$ is a manifold of the same type. The boundary satisfies $\partial P=\pi^{-1}(\partial \mathbf{W})$.

Definition 1 For $q \in \mathrm{W}$, the point $q=\pi(p)$ is said to be the carrier of $p$.

Motion flow and cusps. Define a one-parameter group of transformations $\left\{\mathcal{F}^{t}\right\}$ on $\mathbf{P}$. Let $\mathcal{F}^{t}(p)$ be the configuration $t$ seconds after the car moved from point $p=(q, \phi) \in \mathbf{P}$ (with a unit speed). We call $\mathcal{F}^{t}$ the motion flow over distance $t$; it defines uniquely the car motion along the trajectory. That is, $\mathcal{F}^{t}$ is a piece of curve, a function of $t$. Motion flow $\mathcal{F}^{t}$ can be defined by a combination of elementary motions - rotation to the left, $L^{t}$, rotation to the right, $R^{t}$ (both along a circle of radius $\rho \geq \rho_{\text {min }}$ ), 
and straight line motion $S^{t}:\left\{\mathcal{F}^{t}\right\}=\left\{L^{t}, R^{t}, S^{t}\right\}$, with the elementary transformations defined by

$$
\begin{array}{r}
L^{t}(x, y, \phi)=(x+\rho \sin (\phi+t)-\rho \sin \phi, \\
y-\rho \cos (\phi+t)+\rho \cos \phi, \phi+t) \\
R^{t}(x, y, \phi)=(x-\rho \sin (\phi-t)+\rho \sin \phi, \\
y+\rho \cos (\phi-t)-\rho \cos \phi, \phi-t) \\
S^{t}(x, y, \phi)=(x+t \cos \phi, y+t \sin \phi, \phi)
\end{array}
$$

Given a configuration $p$, we are interested in two cases associated with the flow $\mathcal{F}^{t}(p)$ : (i) flow $\mathcal{F}^{t}$ does not intersect the boundary $\partial \mathbf{W}$; (ii) for some value $t$, the end point of flow $\mathcal{F}^{t}$ is located at the boundary $\Gamma=\partial \mathbf{W}$.

In terms of motion control, case (i) presents two options: (a) reverse the motion (thus producing a cusp), and (b) continue the smooth flow under the group of transformations $\left\{\mathcal{F}^{t}\right\}$ (arcs $q_{1} q_{2}$ and $q_{2} q_{3}$, Figure 1). Case (ii) presents one choice only - reverse the motion in order to stay within $\mathbf{W}$ (positions $q_{1}, q_{2}, q_{3}$, and $q_{4}$, Figure 1 ).

Reversals can take place at the boundary $\partial W$ or inside W. At cusp points, the natural projection $\pi: \mathbf{P} \rightarrow \mathbf{W}$ possesses an involution (reflection) property: that is, a point $p^{-}=\left(q, v^{-}\right) \in \mathbf{P}$ is mapped into the point $p^{+}=$ $\left(q, v^{+}\right) \in \mathbf{P}$, with the same carrier $q$ and opposite velocity vector $v^{+}=-v^{-}$. The behavior of flow $\mathcal{F}^{t}(p)$ in the vicinity of a cusp point $p$ is defined by the left and right limits, $\mathcal{F}^{t}(p) \stackrel{\text { def }}{=} \lim _{t \rightarrow t^{\prime}+0} \mathcal{F}^{t^{\prime}}(p)$ and $\mathcal{F}^{t}(p) \stackrel{\text { def }}{=} \lim _{t \rightarrow t^{\prime}-0} \mathcal{F}^{t^{\prime}}(p)$.

\section{THE BASIC TRANSFORMATION}

\section{A. Reflective Unfolding operator}

Consider a motion flow consisting of two segments, $\gamma_{i}(t)$ and $\gamma_{i+1}(t)$, Figure 2. As parameter $t$ increases, the flow continues from segment $\gamma_{i}(t)$ to segment $\gamma_{i+1}(t)$. When switching from $\gamma_{i}(t)$ to $\gamma_{i+1}(t)$, the car reverses the direction of its motion.

Segment $\gamma_{i}(t)$ starts in configuration $p_{i}$, with carrier $q_{i}=\pi\left(p_{i}\right)$, and ends in configuration $p_{i+1}$, with carrier $q_{i+1}=\pi\left(p_{i+1}\right)$. Carrier $q_{i+1}$ is a cusp point; it belongs to the interior of the domain $\mathbf{W}$ or boundary $\partial \mathbf{W}$. At the cusp, define two configurations, $p_{i+1}^{-}$and $p_{i+1}^{+}$, with the same carrier $q_{i+1}$ and tangents of opposite signs, $v_{i+1}^{-}=$ $-v_{i+1}^{+}$. Segment $\gamma_{i+1}(t)$ starts in $p_{i+1}$ and ends in $p_{i+2}$.

To eliminate the cusp at $q_{i+1}$, we introduce a mapping transformation called the Reflective Unfolding operator, denoted by $\sigma$ :

Definition 2 Mapping $\sigma_{q_{i+1}}$, called Reflective Unfolding (RU) operator with respect to the carrier $q_{i+1}$, is as follows: for any two neighboring subpaths $\gamma_{i}$ and $\gamma_{i+1}$ separated by a cusp $q_{i+1}$, subpath $\gamma_{i}$ is fixed in $\mathbb{R}^{2}$, and subpath $\gamma_{i+1}$ is transformed into an equivalent subpath $\gamma_{i+1}^{(1)}$ by a

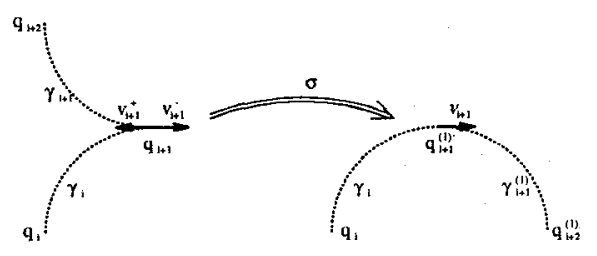

Fig. 2. The $R U$-operator $\sigma$ eliminates a cusp at the carrier $q_{i+1}$ and maps segment $\gamma_{i+1}$ into segment $\gamma_{i+1}^{(1)}$.

$180^{\circ}$ rotation with respect to the carrier $q_{i+1}$; this is denoted as $\sigma_{q_{i+1}}: \gamma_{i} \uplus_{q_{i}} \gamma_{i+1} \rightarrow \gamma_{i} \uplus \gamma_{i+1}^{(1)}$

Here notation $\uplus_{q_{i}}$ on the left of the arrow refers to the original path, with subpaths $\gamma_{i}$ and $\gamma_{i+1}$ joined by a cusp at carrier $q_{i+1}$, and $\uplus$ on the right of the arrow refers to the path obtained after a single application of the RUoperator. (Here and below, superscripts "(1)", "(2)" etc., when used, indicate the number of applications of RUoperator to the corresponding entity). Thus, the operator does the "unfolding" via a simple central symmetry reflection with respect to the cusp point (see Figure 2).

Next we consider some properties of the RU-operator. Recall that a path is not differentiable at a cusp point: $\exists \lim _{t^{\prime} \rightarrow t-0} \mathcal{F}^{t^{\prime}}(p)$ and $\exists \lim _{t^{\prime} \rightarrow t+0} \mathcal{F}^{t^{\prime}}(p)$ in the carrier $q_{i+1}$, but $\lim _{t^{\prime} \rightarrow t-0} \mathcal{F}^{t^{\prime}}(p) \neq \lim _{t^{\prime} \rightarrow t+0} \mathcal{F}^{t^{\prime}}(p)$.

Proposition $1 A$ single application of the $R U$-operator at a cusp point eliminates the cusp.

That is, after one application of RU-operator the path becomes differentiable at the former cusp point. Indeed, configurations $p_{i+1}^{-}$and $p_{i+1}^{+}$have the same carrier $q_{i+1}$ and tangents of opposite signs, $v_{i+1}^{-}=-v_{i+1}^{+}$. After the application of RU-operator, $q_{i+1}$ and $v_{i+1}^{-}$stay the same, and $v_{i+1}^{+}$becomes collinear with $v_{i+1}^{-}$. Equivalently, at carrier $q_{i+1}\left(t^{\prime}\right), \quad \exists \lim _{t^{\prime} \rightarrow t-0} \mathcal{F}^{t^{\prime}}(p)$ and $\exists \lim _{t^{\prime} \rightarrow t+0} \mathcal{F}^{t^{\prime}}(p)$, and, in addition, $\lim _{t^{\prime} \rightarrow t-0} \mathcal{F}^{t^{\prime}}(p)=\lim _{t^{\prime} \rightarrow t+0} \mathcal{F}^{t^{\prime}}(p)$, which guarantees that the flow $\mathcal{F}^{\prime}(p)$ is smooth in $q_{i+1}$.

\section{B. Composition of transformations}

Consider a path $\gamma(t)$ consisting of $n+1$ smooth segments $\gamma_{i}, i=1, \ldots, n+1, \gamma(t)=\gamma_{1} \uplus_{q_{1}} \gamma_{2} \cdots \gamma_{n} \uplus_{q_{n}} \gamma_{n+1}$, connected by $n$ cusps, $q_{1}, \cdots, q_{n}$. We will now apply the RU-operator sequentially to this path, as explained below, obtaining a smooth path as a result.

\section{Definition 3 The composition transformation}

$\sigma_{\left\{q_{1}, \ldots, q_{n}\right\}}=\left\{\sigma_{q_{1}}, \sigma_{q_{2}}, \ldots, \sigma_{q_{n-1}}, \sigma_{q_{n}}\right\}$ of a path $\gamma(t)=$ $\gamma_{1} \uplus_{q_{1}} \gamma_{2} \cdots \gamma_{n} \uplus_{q_{n}} \gamma_{n+1}$ with $n$ cusps is obtained by sequentially applying the Reflective Unfolding operator $n$ times to 
the path, starting with the first cusp $q_{1}$ and proceeding to the last cusp $q_{n}$.

Proposition 2 When applied to a path $\gamma(t)=\gamma_{1} \uplus_{q_{1}}$ $\gamma_{2} \cdots \gamma_{n} \uplus_{q_{n}} \gamma_{n+1}$ with $n$ cusps, the composition transformation $\sigma_{\left\{q_{1}, \ldots, q_{n}\right\}}$ produces a smooth path $\gamma_{1} \uplus \gamma_{2}^{(1)} \uplus \cdots \uplus$ $\gamma_{n}^{(n-1)} \uplus \gamma_{n+1}^{(n)}$

The composition transformation proceeds as follows. First (transformation $\sigma_{q_{1}}$ ), the RU-operator is applied to two subpaths, $\gamma_{1}$ and $\left\{\gamma_{2}, \cdots, \gamma_{n+1}\right\}$, connected by a cusp at carrier $q_{1}$. In this operation, subpath $\gamma_{1}$ is fixed, and subpath $\left\{\gamma_{2}, \cdots, \gamma_{n+1}\right\}$ is rotated $180^{\circ}$, resulting in a path that is smooth at $q_{1}$,

$$
\begin{array}{cc}
\sigma_{q_{1}}: & \gamma_{1} \uplus_{q_{1}}\left\{\gamma_{2} \uplus_{q_{2}} \gamma_{3} \cdots \gamma_{n} \uplus_{q_{n}} \gamma_{n+1}\right\} \rightarrow \\
& \gamma_{1} \uplus \gamma_{2}^{(1)} \uplus_{q_{2}} \gamma_{3}^{(1)} \cdots \gamma_{n}^{(1)} \uplus_{q_{n}} \gamma_{n+1}^{(1)}
\end{array}
$$

Then RU-operator is applied again (transformation $\left.\sigma_{q_{2}}\right)$, this time at the second cusp, to two subpaths, $\left\{\gamma_{1}, \gamma_{2}^{(1)}\right\}$ and $\left\{\gamma_{3}^{(1)}, \cdots, \gamma_{n+1}^{(1)}\right\}$, thus eliminating the cusp at $q_{2}, \gamma_{1} \uplus \gamma_{2}^{(1)} \uplus \gamma_{3}^{(2)} \uplus_{q_{3}} \gamma_{4}^{(2)} \cdots \gamma_{n}^{(2)} \uplus_{q_{n}} \gamma_{n+1}^{(2)}$. By continuing this process through the last cusp, obtain Proposition 2.

\section{MOVING IN A LIMITED DOMAIN: CONTROL STRATEGY}

Here we develop the control strategy for finding the optimal (curvature-constrained) path within the workspace $D$ (see the problem statement in Section II). The strategy makes use of the composite transformation described above. We conclude the section by showing that this strategy produces paths that are the shortest possible and of the lowest complexity (i.e. have the smallest number of cusps). It is also shown that there exist infinitely many paths of the same length but higher complexity, and infinitely many paths of the same lowest complexity but longer.

The strategy makes use of two simple considerations: (a) each path segment is an arc of a circle of minimum radius; (b) to use the space most effectively, each path segment originates and ends at the boundary of disc $D\left(q_{0}, R\right)$ (we call this boundary-to-boundary motion). In the resulting path, the car will alternate between forward and reverse motion, with reversal cusps at the disc boundary, until the final configuration is reached.

\section{A. The equivalent problem}

A typical path by a car in the disc $D\left(q_{0}, R\right)$ is shown in Figure 1. Starting at the configuration $p_{0}=\left(q_{0}, \phi_{0}\right)$, the car first moves along an arc of radius $\rho_{\text {min }}$, and reaches the boundary of the workspace $\mathrm{W}=D\left(q_{0}, R\right)$ at point $q_{1} \in \partial \mathbf{W}$, with orientation $\phi_{1}^{-}$, or equivalently, with the

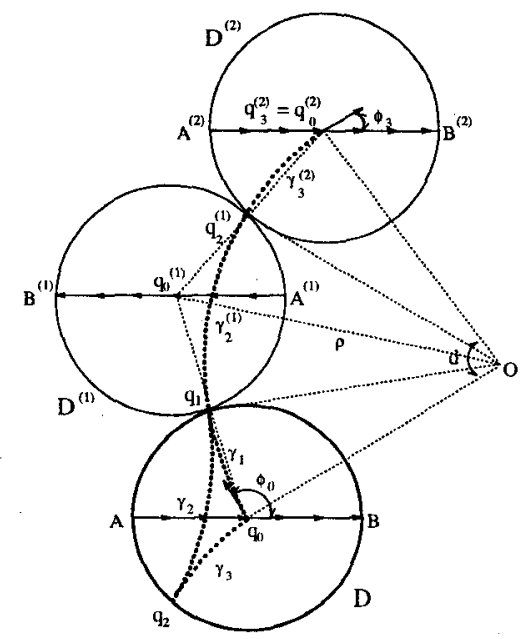

Fig. 3. The mapping is defined as a central symmetry with respect to the reflection point; the central symmetry with respect to point $q_{1}$ maps $q_{2}$ into $q_{2}^{(1)}, A$ into $A^{(1)}$, and $B$ into $B^{(1)}$; disc $D$ into the disc $D^{(1)}$.

unit velocity vector $v_{1}^{-}$. Then the car reverses and moves backward along the second arc segment, with the initial velocity vector $v_{1}^{+}$, reaching $\partial \mathbf{W}$ at point $q_{2}$, with the orientation $v_{2}^{-}$. The path from $p_{0}=\left(q_{0}, \phi_{0}\right)$ to $p_{2}=$ $\left(q_{2}, \phi_{2}\right)$ thus contains two arcs, $\gamma_{1}$ and $\gamma_{2}$, connected by a cusp with tangency $q_{1}$ (Figure 3 ).

By applying mapping (RU-operator) $\sigma_{q_{1}}$ to the path $\left\{\gamma_{1}, \gamma_{2}\right\}$, a smooth path $\left\{\gamma_{1}, \gamma_{2}^{(1)}\right\}$ is obtained. Similarly, by applying mapping $\sigma_{q_{2}}$ to subpaths $\left\{\gamma_{1}, \gamma_{2}^{(1)}\right\}$ and $\gamma_{3}^{(1)}$ connected by cusp $q_{2}^{(1)}$, obtain the path $\left\{\gamma_{1}, \gamma_{2}^{(1)}, \gamma_{3}^{(2)}\right\}$. The following geometric fact holds:

Proposition 3 Given the disc $D\left(q_{0}, R\right)$ and the starting point at its center, any path based on boundary-toboundary motion and consisting of arcs of fixed radius has its every odd arc segment pass through the center of $D$.

To see this, consider an equivalent problem (Figure 3 ). Apply mapping $\sigma_{q_{1}}$ followed by the mapping $\sigma_{q_{2}}$. Now, the motion flow $q_{0} \rightarrow q_{1} \rightarrow q_{2} \rightarrow q_{0}$ within the disc $D\left(q_{0}, R\right)$ is equivalent to the flow $q_{0} \rightarrow q_{1} \rightarrow q_{2}^{(1)} \rightarrow q_{0}^{(2)}$ within the union of discs $D \cup D^{(1)} \cup D^{(2)}$. The resulting motion will be along the circle of radius $\rho$ centered at point $O$. After transformation $\sigma_{q_{1}}$, the center $q_{0}$ of disc $D$ maps into the center $q_{0}^{(1)}$ of disc $D^{(1)}$. Transformation $\sigma_{q_{2}}^{(1)}$ maps the center $q_{0}^{(1)}$ of disc $D^{(1)}$ into the center $q_{0}^{(2)}$ of disc $D^{(2)}$. It also follows that $\left|q_{0} q_{0}^{(1)}\right|=\left|q_{0}^{(1)} q_{0}^{(2)}\right|=2 R$, and points $q_{0}, q_{1}$, and $q_{2}^{(1)}$ belong to the same circle $(O, \rho)$. This means that point $q_{0}^{(2)}$ also belongs to the circle $(O, \rho)$.

To summarize, there are two useful facts about the boundary-to-boundary motion flow within a disc: 


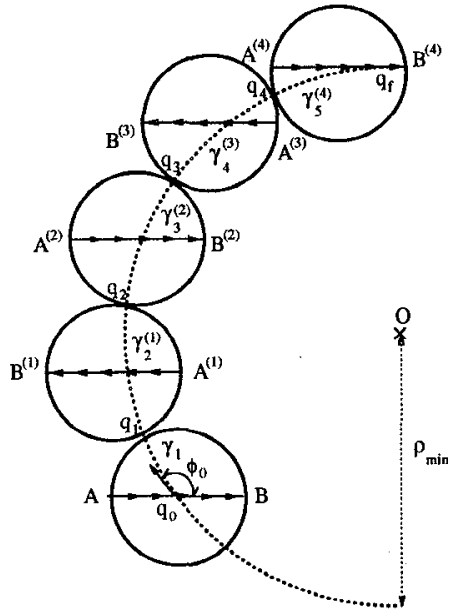

Fig. 4. Motion with reversals within the workspace $\mathbf{W}=$ $D\left(q_{0}, R\right)$ is equivalent to the motion along a circle of radius $\rho_{\min }$ centered at point $O$.

(1) The motion flow is periodic. If the motion starts at the disc center, then after two reflections from the boundary the flow will pass through the disc center again.

(2) The original motion flow can be mapped into an equivalent motion flow along a circle and without cusps.

It suggests another formulation of the problem at hand:

The equivalent problem: Given the car's initial configuration in the plane, find a curvature-constrained smooth trajectory (of curvature $1 / \rho_{\min }$ ) leading to the final car orientation.

Without loss of generality, assume a positive orientation of the reference axis at the final orientation. One can see already that the equivalent problem is tantamount to covering the equivalent trajectory (an arc of radius $\rho_{m i n}$ ) by discs of radius $R$ following mapping rules. Once this is done, the arc segments of the equivalent path can be "folded back" into the actual workspace $\mathbf{W}=D\left(q_{0}, R\right)$ to complete the construction of the trajectory. Below we study the equivalent problem in more detail.

\section{B. Cusp elimination}

Consider the sequence $\left(a_{i}\right), i=0,1,2, \ldots$ of orientations which correspond to those arc segments that pass through the center of disc $D$. According to Proposition 3, $a_{0}=\phi_{0}$ is the initial orientation of the car, $a_{1}=\phi_{3}, a_{2}=\phi_{5}$ etc. Denote by $d$ the common difference $a_{1}-a_{0}$. Then the $j$-th term of the sequence $\left(a_{i}\right)$ is defined as $a_{j}=a_{1}+(j-1) d$, and the common difference $d$ is defined as (see Figure 5)

$$
d=2 \angle q_{0} O q_{1}+2 \angle q_{1} O_{2} q_{0}=2 \theta_{1}+2 \theta_{2}
$$

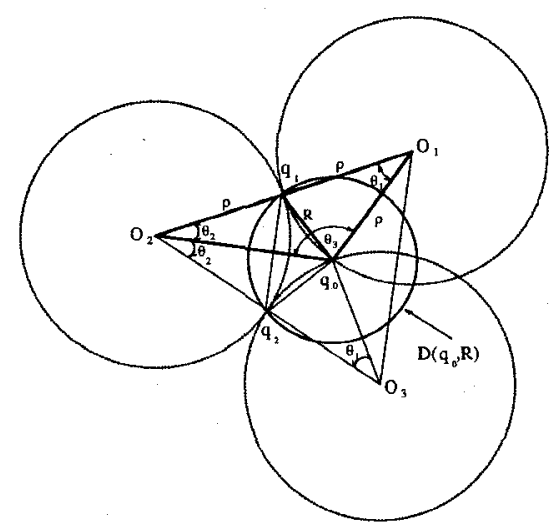

Fig. 5. The car is restricted to move within the disc $D\left(q_{0}, R\right)$ of the radius $R$ centered at point $q_{0}$. The car trajectory is from point $q_{0}$ to $q_{1}$ to $q_{2}$ to $q_{0}$, along the arcs of circles $\left(\mathrm{O}_{1}, \rho\right),\left(\mathrm{O}_{2}, \rho\right)$, and $\left(\mathrm{O}_{3}, \rho\right)$, respectively.

From Figure 3, angle $\theta_{1}$ is found as

$$
\theta_{1}=2 \arcsin \frac{R}{2 \rho}
$$

Considering of the triangle $\triangle \mathrm{O}_{2} \mathrm{O}_{1} q_{0}$, obtain the expression for angle $\theta_{2}$ :

$$
\theta_{2}=\arcsin \left\{\frac{R}{2} \sqrt{\frac{4 \rho^{2}-R^{2}}{\rho^{2}+2 R^{2}}}\right\}
$$

Substituting the expressions (6) and (7) for $\theta_{1}$ and $\theta_{2}$ into (5), the common difference $d$ is found as

$$
d=4 \arcsin \left\{\frac{R}{2 \rho}\right\}+2 \arcsin \left\{\frac{R}{2} \sqrt{\frac{4 \rho^{2}-R^{2}}{\rho^{2}+2 R^{2}}}\right\}
$$

Define a positive number $\varepsilon>0$ as

$$
\begin{aligned}
& \varepsilon= \begin{cases}\phi_{0}-k d, & \text { if } \phi_{0} \leq 180^{\circ}, \\
\phi_{0}+k d, & \text { if } \phi_{0} \geq 180^{\circ}, \text { where }\end{cases} \\
& k= \begin{cases}\left\lfloor\frac{\phi_{0}}{d}\right\rfloor, & \text { if } \phi_{0} \leq 180^{\circ}, \\
\left\lfloor\frac{360^{\circ}-\phi_{0}}{d}\right\rfloor, & \text { if } \phi_{0} \geq 180^{\circ}\end{cases}
\end{aligned}
$$

and symbol $\lfloor x\rfloor$ denotes the largest integer less or equal to $x$.

Recall the task at hand (Figure 1): Given the car's initial and final configurations, $p_{0}=\left(0,0, \phi_{0}\right)$ and $p_{f}=$ $(0,0,0)$, find the shortest path for the car's center of mass $C$ that is confined to the disc $D\left(q_{0}, R\right)$. We can now formulate the algorithm for solving this problem:

Step 1: Find from (9) the maximum integer $k$. If $\phi_{0} \leq$ $180^{\circ}$, move forward clockwise (or, if $\phi_{0} \geq 180^{\circ}$, move backward counterclockwise) along an arc of radius $\rho_{\min }$, up to the boundary of disc $D$. Using the reflection rule, reverse the direction of motion. Repeat this pattern $2 k$ times and arrive at the center $q_{0}$ of disc $D\left(q_{0}, R\right)$; at this point, the 
orientation angle is $\varepsilon$.

Step 2: The last two reversals take place at the circle of $\overline{\operatorname{radius}}_{l}$ concentric with disc $D\left(q_{0}, R\right)$. To find $R_{l}$, substitute $\varepsilon\left(\bmod 180^{\circ}\right)$ instead of $d$ into equation (8), solve equation (8) for $R$, and take $R_{l}=R$.

\section{Optimality of the control strategy}

We now show that the proposed control strategy guarantees the shortest path possible between the initial and final configurations. In addition, this path is of the lowest complexity possible.

Proposition 4 The motion flow obtained by the control strategy is a geodesic path.

To prove this statement, we use the following auxiliary proposition, which is geometrically obvious and is stated without proof.

Proposition 5 (auxiliary) Consider three configurations, $p_{0}=\left(M_{0}, \alpha_{0}\right), p_{1}=\left(M_{1}, \alpha_{1}\right), p_{2}=\left(M_{0}, \alpha_{2}\right)$. Configuration $p_{0}$ is connected with $p_{1}$ by an arc $\eta_{1}$ of radius $R_{1}$, and with $p_{2}$ by an arc $\eta_{2}$ of radius $R_{2}$. Assume $R_{2}>R_{1}$ and $\alpha_{1}=\alpha_{2}$. Then, the length of arc $\eta_{2}$ is grater than that of arc $\eta_{1}$.

Note that there are infinitely many paths that would bring the car from its initial to its final orientation. Indeed, since the rate of orientation change is proportional to the radius of curvature and to the length of the segment traveled, any path that includes at least one arc segment of radius larger than $\rho_{\min }$ is longer than the one whith the corresponding arc being of radius $\rho_{m i n}$ (Proposition 5). This observation shows that the path produced by the proposed algorithm is indeed the shortest one, which completes the proof of Proposition 4.

Proposition 6 The algorithm guarantees that the path produced is of the lowest possible complexity, i.e., it contains the minimum number of motion reversals.

To see this, think of the problem at hand as that of folding an arc of a circle of radius $\rho_{\text {min }}$ (i.e. the equivalent path) back into the workspace disc of radius $R$. That operation is analogous to covering the said arc by discs $D, D^{(1)}, D^{(2)}, \ldots$ of radius $R$ according to the mapping rule defined by the Reflective Unfolding operator. The more discs are needed to cover the entire arc, the higher is the complexity of the path; this is because the boundary-toboundary motion makes the number of discs correspond to the number of reversals. Since the discs are of the maximum possible radius, fewer discs are required.

The above analysis of the equivalent problem leads to two other interesting conclusions:
Proposition 7 There are infinitely many geodesics of the same length, but of higher complexity.

Covering the same equivalent (geodesic) path by discs of a smaller radius leads to a larger number of discs - that is, to more motion reversals and higher complexity of the actual path.

Proposition 8 There are infinitely many paths of the lowest complexity, all of bounded length.

This can be easily seen on a simple example where all path segments, except the last one, are those of the optimal path. Note that the last segment can be chosen as an arc of radius larger than $\rho_{\text {min }}$ - thus producing an infinite number of possibilities. The length of such segments will be bounded from below by the geodesic path and from above by $k d+\rho \varepsilon$, as defined in (8) and (9), where $\rho$ is a solution of (8) with $d$ taken equal to $\varepsilon$.

\section{CONCLUSION}

This paper proposes an approach for solving the problems of motion planning for a nonholonomic system operating within a limited two-dimensional domain. The approach is demonstrated on a control strategy for a car moving within a disc. The idea of trajectory unfolding allows one to obtain the optimal solution in an analytic form. The optimality of paths produced by the approach is two-fold: each path is of the shortest length possible and of the lowest complexity possible.

\section{REFERENCES}

[1] J. A. Reeds and L. A. Shepp. Optimal paths for a car that goes both forwards and backwards. Pacific Journal of Mathematics, 145:367-393, 1990.

[2] J. Laumond. "Feasible trajectories for mobile robots with kinematic and environment constraints". Elsiver Science Publishers B.V., Amsterdam, The Netherlands, pp. 346-354, 1986. Preprints of the international conference on intelligent autonomous systems.

[3] S. Fortune and G. Wilfong. Planning constrained motion. 20th Proc. ACM Symp. on Theory of Computing, pages 445-459, May 1988. Chicago, IL.

[4] L. E. Dubins. On curves of minimal length with a constraint on everage curvature, and with prescribed initial and terminal positions and tangents. American Journal of Mathematics, 79:497-516, 1957.

[5] A. Shkel and V. Lumelsky. The Jogger's Problem: Accounting for body dynamics in real-time motion planning. IEEE/RSJ Intern. Workshop On Intelligent Robots and Systems, August 1995. Pittsburgh, PA. 